\title{
GOSSIPING WITH UNIT MESSAGES IN KNOWN RADIO NETWORKS
}

\author{
Leszek Gassieniec* and Igor Potapov \\ Department of Computer Science, University of Liverpool \\ Liverpool L69 7ZF, UK. \\ leszek@csc.liv.ac.uk, igor@csc.liv.ac.uk
}

\begin{abstract}
A gossiping is a communication primitive in which each node of the network possesses a unique message that is to be communicated to all other nodes in the network. We study the gossiping problem in known ad hoc radio networks, where during each transmission only unit messages originated at any node of the network can be transmitted successfully. We survey a number of radio network topologies. Assuming that the size (a number of nodes) of the network is $n$ we show that the exact complexity of radio gossiping in stars is $2 n-1$, in rings is $2 n \pm O(1)$, and on a line of processors is $3 n \pm O(1)$. We later prove that radio gossiping in free trees is harder and it requires at least $3 \frac{1}{6} n-16$ time steps to be completed. For free trees we also show a gossiping algorithm with time complexity $5 n+8$. In conclusion we prove that in general graphs radio gossiping requires $\Omega(n \log n)$ time, and we propose radio gossiping algorithm that works in time $O\left(n \log ^{2} n\right)$.
\end{abstract}

\section{Introduction}

The importance of communication networks and their use on a daily basis has been steadily growing over the past few decades. One of the most striking examples of modern networking technology is the Internet with its diverse applications in research, business, education, and entertainment. Mobile radio networks [19] are expected to play an important role in future commercial and military applications. These networks are suitable in situations where instant infrastructure is needed and no central system administration (such as base stations in a cellular system) is available.

There are two important communication primitives used in the process of dissemination of information in networks: broadcasting and gossiping. In the broadcasting problem, a distinguished source node has a message that needs to

*Supported in part by EPSRC grants GR/N09855 and GR/R85921. 
be sent to all other nodes. The gossiping is a communication primitive in which each node of the network possesses a unique message that is to be communicated to all other nodes in the network. The gossiping problem raises naturally both in the theoretical as well as more applied setting. It is a part of several multiprocessor computation tasks, such as global processor synchronization, linear system solving, Discrete Fourier Transform, and parallel sorting, e.g.,see [4, 13].

Most of the work in the field has been done under the assumption that processors can transmit messages of an arbitrary size in a single time step. The gossiping problem with bounded (size) messages was previously studied in the matching model, e.g., see [3]. In this model during every time step nodes organize themselves and exchange information in independent pairs. The results presented in [3] include the study of the exact complexity of the gossiping problem in Hamiltonian graphs and $k$-ary trees, and optimal asymptotic bounds for general graphs, in the matching model with unit messages. Their paper contains also a number of asymptotically optimal results in the matching model with messages of arbitrarily bounded size. Another interesting study of the gossiping problem with limited size messages in graphs with bounded degree can be also find in [12].

In this paper we study the gossiping problem with unit messages in known ad hoc radio networks. We adopt here a communication model used previously, e.g., in $[1,14,6]$. A radio network is modeled as an undirected graph $G=(V, E)$. The nodes in set $V=\left\{v_{0}, . ., v_{n-1}\right\}$ are interpreted as processors (transmitter/receiver devices) while undirected edges in set $E$ indicate that every neighboring node in the graph is in the transmission range. The processors work synchronously. In each time step, any processor can either transmit or receive a message. A message transmitted by processor $v$ reaches all its neighbors in the same time step. However, any neighbor $w$ can receive it only if no message from another processor reaches it at this time step. Otherwise a collision occurs and none of the messages is delivered to $w$. The size of the network corresponds to the number of nodes in the underlying graph of connections. In what follows we assume that the size of the network is $n$.

It is only recently that studies on radio gossiping have been intensified, see $[7,9,10,15,11,16,17,18]$. However this work is devoted to the case when the messages used in the gossiping process can be of an arbitrary size. Under this strong assumption Chrobak et al. [9] showed that deterministic gossiping can be performed in unknown directed ad-hoc radio networks in time $O\left(n^{3 / 2} \log ^{2} n\right)$. A constructive version of their algorithm was recently proposed by Indyk, see [16]. This result was recently improved by Gasieniec and Lingas [15] for networks with diameter $D=n^{\alpha}$, for $\alpha<1$. They presented an alternative gossiping algorithm working in time $O\left(n \sqrt{D} \log ^{2} n\right)$. These results show that radio networks with a long diameter constitute a bottleneck in deterministic radio gossiping with messages of an arbitrary size. An alternative approach to the radio gossiping problem was presented by Clementi et al. in [11]. They proposed deterministic gossiping algorithm with running time $O\left(D d^{2} \log ^{3} n\right)$, where $d$ stands for the maximum in-degree of the underlying graph of con- 
nections. Chrobak et al. in [10] proposed also a randomized radio gossiping algorithm with expected running time $O\left(n \log ^{4} n\right)$. A study on oblivious gossiping in ad hoc radio networks can be found in [7]. An alternative radio model was studied by Ravishankar and Singh. They presented distributed gossiping algorithms for networks with nodes placed randomly on a line [17] and a ring [18].

In this paper we initiate a discussion on gossiping in known radio networks with messages of a limited size. In what follows we assume that the messages originated in all nodes of the network are unique and they are of the same size. Moreover each transmission performed by any node of the network can contain only one, a unit, message originated in some node of the network. A similar concept of communication in unknown radio networks with messages of a limited size has been recently adopted by Christersson et al. in [8]. Another interesting study of randomized multiple communication in unknown radio networks with messages limited to $O(\log n)$ bits can be found in [2].

\section{Radio gossiping in stars and rings}

A star of size $n$ is a free tree $S=\{V, E\}$, where $V=\left\{v_{0}, . ., v_{n-1}\right\}$ and $E=\left\{\left(v_{0}, v_{1}\right), . .,\left(v_{0}, v_{n-1}\right)\right\}$. Node $v_{0}$ is called a central node (center) and all other nodes form arms of the star.

Theorem 1 The exact complexity of radio gossiping in stars of size $n$ is $2 n-1$.

A ring of size $n$ is a graph $R=(V, E)$, s.t., $V=\left\{v_{0}, . ., v_{n-1}\right\}$ and $E=$ $\left\{\left(v_{i}, v_{(i+1) \bmod n}\right): i=0, . . n-1\right\}$.

Theorem 2 The exact complexity of radio gossiping in rings of size $n$ is $2 n \pm$ $O(1)$.

Lower bound As in other instances of the gossiping problem also in this case every unit message has to be transmitted to all other nodes of the network. We say that a delivery occurs at node $v$ at time step $t$ if node $v$ receives a message at time step $t$. We show that during a single time step any communication algorithm performs at most $\lfloor n / 2\rfloor$ deliveries. Assume opposite, i.e., at least $\lfloor n / 2\rfloor+1$ deliveries have occurred. It means that there exist three nodes with consecutive labels $v_{i-1}, v_{i}, v_{i+1}$ to which some messages have been delivered. However this leads to a contradiction since node $v_{i}$ is not able to receive any messages when its both (and only) neighbors are in the receiving mode. Finally since every node is expecting $n-1$ deliveries the total number of steps required in this case is $\geq n(n-1) /\lfloor n / 2\rfloor \geq 2 n-2$.

Upper bound We show that there exists an algorithm performing radio gossiping on a ring of size $n$ in time $2 n+9$. The algorithm consists of a number of steps. During each step consecutive processors along the ring are grouped into alternating pairs, i.e., active (transmitting) pairs alternated with dormant (expecting messages) pairs. We place as many as possible alternating pairs on 
the ring assuring that all active pairs are at least at distance 2 apart. Note that in the worst case at one point on the ring there may be two active pairs at distance 5 apart (if the distance was six we could introduce another active pair). We call this phenomenon as a gap, see Figure 1b. The pattern of al-
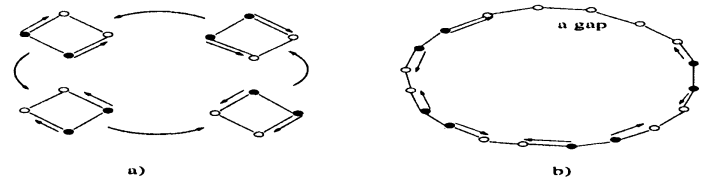

Figure $1 . \quad$ a) 4 consecutive rounds, b) maximum gap

ternating pairs is fixed however it is rotated along the ring by one position, e.g., in clockwise order at the end of each step. Note that two elements of any active pair are responsible for transmissions to opposite (clockwise and anticlockwise) directions. A round for a node is a number of steps between two consecutive clockwise transmissions. If the gap was of size 2 ( $n$ is a multiple of 4) all rounds would consist of 4 steps, see Figure 1a. However some long rounds can have as many as 7 steps when the gap is of size 5 . Since every original message has to be transmitted through at most $\lceil(n-1) / 2\rceil \leq n / 2$ nodes in clockwise (and anti-clockwise) order and each long round occurs with periodicity at least $\lceil(n-3) / 4\rceil \geq n / 4-1$, each traversing message can experience at most 3 long rounds. Thus the time complexity of radio gossiping in rings of size $n$ is bounded by $4 \cdot n / 2+3 \cdot 3=2 n+9$.

\section{Radio gossiping on a line}

A line of size $n$ is a free tree $L=\{V, E\}$, where $V=\left\{v_{0}, . ., v_{n-1}\right\}$ and $E=\left\{\left(v_{i}, v_{i+1}\right): i=0, . . n-2\right\}$. Nodes $v_{0}$ and $v_{n-1}$ are called the left end and the right end respectively.

Theorem 3 The exact complexity of radio gossiping on a line of size $n$ is $3 n \pm O(1)$.

Lower bound To prove the lower bound $3 n-O(1)$ we will consider a similar but easier problem called here a monotonic gossiping. The task is to send all original messages only to the nodes being on their right hand side. We will need the following definitions.

Stack-and-knot problem A stack and knot $S K(n)$ is an object that consists of a short line of length 4 , a knot $K$, and a stack $S$ of $n$ messages available at node $v_{0}$, see Figure 2a. A stack-and-knot problem is to move all messages from the stack at node $v_{0}$ to node $v_{3}$ using radio transmissions with unit messages.

Lemma 1 The exact complexity of stack-and-knot problem in $S K(n)$ is $3 n$. 
a)

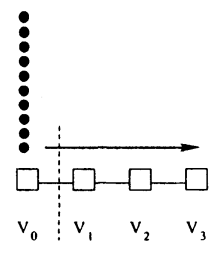

b)

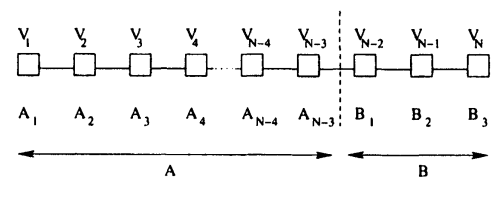

Figure 2. a) Gossiping in part $B$; b) Line $L$ of $n$ nodes

Proof: Due to a collision problem during each step of any algorithm we can perform at most one successful transmission towards the right end of the knot. Thus the process of transmission of $n$ messages from stack $S$ at node $v_{0}$ to node $v_{4}$ requires at least $3 n$ independent steps.

Note also that the algorithm with transmission pattern, s.t., node $v_{i}$ transmits in time step $t$ if $(i=t) \bmod 3$, where initial time step $t=0$, solves stack-and-knot problem in $S K(n)$ in time $3 n$.

Lemma 2 Monotonic gossiping on a line of size $n$ requires $3 n-6$ steps.

Proof: Let $L$ be a line of size $n$. Lets split line $L$ into two parts $A$ and $B$, where part $A$ is formed by the left end of $L$ of size $n-3$ and part $B$ corresponds to the right end of size 3 , see Figure $2 \mathrm{~b}$. According to Lemma $1 \mathrm{a}$ transmission of all $(n-3)$ messages from part $A$ to the node $B_{3}$ takes at least $3(n-3)$ steps. Note that to complete monotonic gossiping the additional three steps are required to transmit to $B_{3}$ messages originated in nodes $B_{1}$ and $B_{2}$. It means that to deliver all messages from the nodes of line $L$ to the node $B_{3}$ (which is equivalent to monotonic gossiping) we need at least $3(n-3)+3=3 n-6$ rounds.

Corollary 1 Radio gossiping on a line of size $n$ requires $3 n-6$ steps.

Lemma 3 Monotonic gossiping on a line of size $n$ can be completed in $3 n-3$ steps.

Proof: The algorithm runs in rounds. Each round consists of 3 steps. During step $i$, for $i=0,1,2$, all nodes with index $(j=i) \bmod 3$, for $j=0, . ., n-1$, transmit and all other nodes remain silent (expecting messages). Following this pattern during each round every node sends one message towards the right end of the line. And after $n-1$ rounds the monotonic gossiping is completed.

Upper bound We present here a gossiping algorithm with running time $3 n+$ $O(1)$ that is a combination of algorithms on a ring and quick pipelining on a line. However before we start the presentation we give an outline of two simpler algorithms working respectively in $4(n-1)$ and $3 \frac{1}{2} n-3$ steps. Note that applying the idea of alternating pairs (see section 2, the gap problem does not exist here) on a line, each round is of size 4 and the gossiping problem can 
be solved in $4(n-1)$ steps. The efficiency of this algorithm can be improved when we notice that gossiping into two directions is required only during initial $\lfloor n / 2\rfloor$ rounds. Afterwards sequences of messages traversing to the left and to the right become disjoint. This allows to use monotonic gossiping on both of them. Thus the total time of improved gossiping algorithm is bounded $\lfloor n / 2\rfloor \cdot 4+\lfloor n / 2\rfloor \cdot 3-3 \leq 3 \frac{1}{2} n-3$.

In what follows we present further improvement showing that monotonic gossiping is actually performed on a sparser sequence of messages allowing us to achieve a gossiping algorithm with running time $3 n+O(1)$. The gossiping algorithm works in two phases: Phase 1 and Phase 2. The main goal of Phase 1 is to move all messages originated in the left half of $L$ to its right half (as far as possible) and vice versa. During Phase 1 nodes run a code of two different processes. Process 1 is responsible for transmission of original messages in two opposite directions. Initially all nodes and then gradually decreasing number of central nodes are involved in that process. Process 2 is responsible for efficient monotonic gossiping (on a sparse sequence of messages) on both ends of the line. During Phase 2 all nodes take part in efficient monotonic gossiping and they run the code of Process 3.

More formally we define sets of pairs $(i, t)$, where $i$ stands for a label of a node and $t$ is a number of a time step, as follows:

- $G_{1}=\left\{(i, t) \mid(0<t \leq 2 n)\right.$ and $\left.\left(r_{1} \leq i \leq n-r_{1}\right)\right\}$,

- $G_{2 L}=\left\{(i, t) \mid(12<t \leq 2 n+12)\right.$ and $\left.\left(i \leq n / 2-\left(r_{1}-3\right)\right)\right\}$,

- $G_{2 R}=\left\{(i, t) \mid(12<t \leq 2 n+12)\right.$ and $\left.\left(i \geq n / 2+\left(r_{1}-3\right)\right)\right\}$,

- $G_{3 L}=\left\{(i, t) \mid(2 n+12<t \leq 3 n+12)\right.$ and $\left.\left(i \leq n / 2-r_{2}\right)\right\}$,

- $G_{3 R}=\left\{(i, t) \mid(2 n+12<t \leq 3 n+12)\right.$ and $\left.\left(i \geq n / 2+r_{2}\right)\right\}$.

Phase 1 corresponds to sets $G_{1}, G_{2 L}$, and $G_{2 R}$, where pairs in set $G_{1}$ represent nodes running the code of Process 1 and pairs in sets $G_{2 L}$ and $G_{2 R}$ represent nodes running the code of Process 2 on the left end and on the right end of a line respectively. Similarly Phase 2 corresponds to sets $G_{3 L}$ and $G_{3 R}$ whose elements represent nodes running the code of Process 3 on the left end and on the right end of a line respectively, see Figure 3a.

Phase 1 and Phase 2 run in rounds. Each round of Phase 1 consists of 4 steps and each round of Phase 2 consists of 2 steps. During Phase 1 a number of a round $r_{1}$ is defined as $\lceil t / 4\rceil$, where $t$ is a current time step. We show later that Phase 1 takes at most $2 n+12$ steps, i.e., $\lfloor n / 2\rfloor+3$ rounds. Similarly, during Phase 2 a number of a round $r_{2}$ corresponds to the value $\lceil(t-(2 n+12)) / 2\rceil$, where $t$ is a current time step.

The nodes governed by Process 1 execute a fixed pattern of transmissions during each round. The pattern is based on values of $(t \bmod 4)$ and $(i \bmod 4)$, where $t$ is a current time step and $i$ is an index of a node. In contrast Process 2 and Process 3 have more complex the transmission selection mechanism that is based on offset values $b, c, d$ and $e$, within sets $G_{*}$, compare with Figure $3 \mathrm{~b}$. The transmission selection in Process 2 is based on values $(b \bmod 3)$ in $G_{2 L}$ and $(c \bmod 3)$ in $G_{2 R}$ as well as the number of an internal step $t \bmod 4$ of 
a)

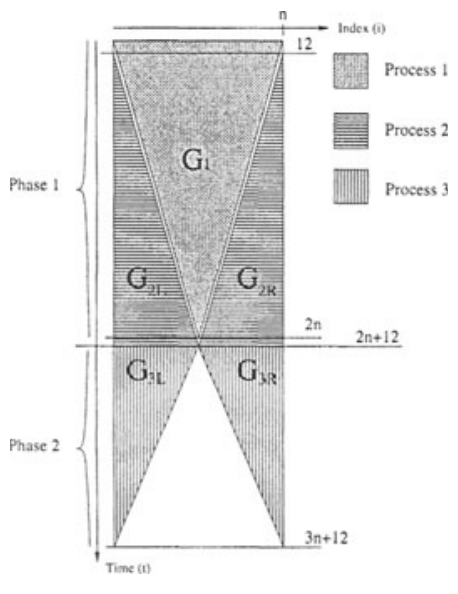

b)

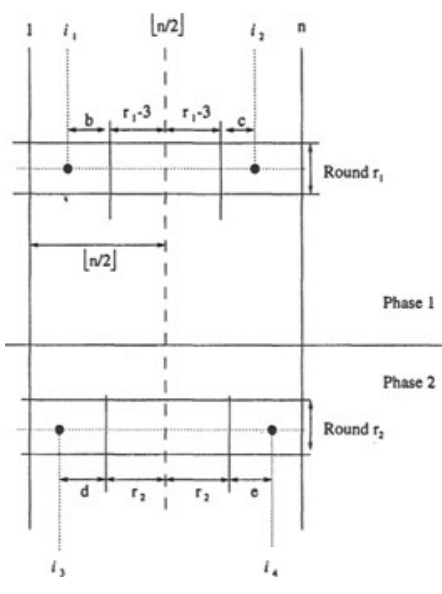

Figure 3. Gossiping on a line: a) Sets $G_{1}, G_{2 L}, G_{2 R}, G_{3 L}$, and $G_{3 R}$; b) Offset calculation.

round $\lceil t / 4\rceil$. Similarly the transmission selection in Process 3 is based on values $(d \bmod 3)$ in $G_{3 L}$ and $(e \bmod 3)$ in $G_{3 R}$ as well as the number of an internal step of round $\lceil(t-(2 n+12)) / 2\rceil$.

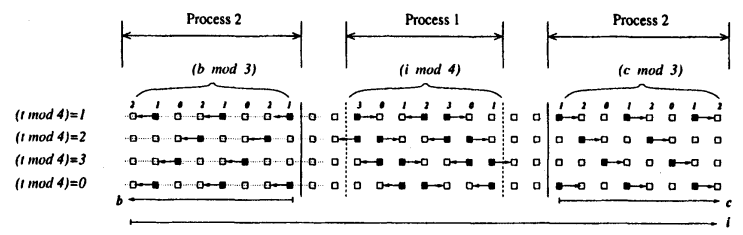

Figure 4. One round of Phase 1

Analysis of Phase 1: Initially each node contains a unit message. This message is kept in two copies. One will be send to the left and the other one to the right neighbour. The following invariant holds. During an execution of one round (four consecutive steps) of Process 1 each node receives two new messages: one from its left and one from its right neighbour. It also transmits two messages: one message to its right neighbour (received during the last round from its left neighbour) and one message to its left neighbour (received during the last round from its right neighbour), see middle part in Figure 4. Note also that shrinking middle part of the line executing code of Process 1 after each round leaves always 2 messages on its left and 2 messages on its right border. These two messages are later dispatched and transmitted to the end of the line by Process 2 .

Both ends of the line are managed by Process 2 as follows. An invariant at the beginning of each round states that every third node is empty (starting with 


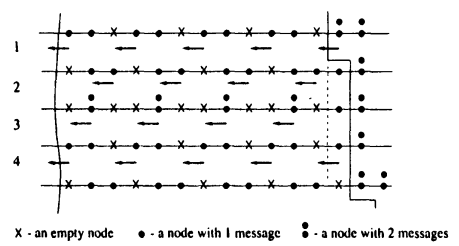

Figure 5. One round of Process 2

the most central one) and all other nodes contain a unit message, see Figure 5. During initial 3 steps of each round every node $\in G_{2 L} \cup G_{2 R}$ receives one message from its right neighbour and sends one message to its left neighbour. The last step of each round is used to move to the left (right) additional one half of messages available at nodes in $G_{2 L}\left(G_{2 R}\right)$. This is to move a pattern of empty nodes towards the center of a line in order to maintain a validity of the invariant at the beginning of the next round. Note also that at any node the execution of Process 2 starts three rounds after the execution of Process 1 is completed in order to avoid collisions caused by nodes executing codes of different processes. In total, Phase 1 consists of $\lfloor n / 2\rfloor+3$ rounds which is $(\lfloor n / 2\rfloor+3) \cdot 4 \leq 2 n+12$ time steps.

Analysis of Phase 2: The order in which messages are positioned on the line $L$ at the end of Phase 1 allows to move them to the end of the line (in each half independently) in time $2 \cdot\lfloor n / 2\rfloor$. This is done by the execution of the algorithm used in the proof of Lemma 1 as follows. In each half of $L$ all messages are grouped into two disjoint sequences $S_{1}$ and $S_{2}$ in which consecutive messages are at distance 3 apart. In odd steps of Phase 2 the monotonic gossiping algorithm is applied on sequence $S_{1}$ and in even steps on sequence $S_{2}$. The monotonic gossiping algorithm applied on each sequence requires (and it can be completed in) $\lfloor n / 2\rfloor$ steps since this is the distance that has to be traversed by the most centrally positioned message and all messages traverse with the speed one position per one time step. Thus the total time of monotone gossiping performed in Phase 2 is bounded by $2 \cdot\lfloor n / 2\rfloor \leq n$.

Lemma 4 Radio gossiping on a line of size $n$ can be performed in time $3 n+12$.

Proof: Phase 1 requires $\leq 2 n+12$ time steps. The partial monotonic gossiping in Phase 2 takes time $\leq n$. Thus the total time complexity is bounded by $3 n+12$.

Theorem 3 follows from Lemma 4 and Corollary 1.

\section{Gossiping in trees}

In this section we discuss the time complexity of the radio gossiping problem in general free trees. 
Theorem 4 The exact complexity of radio gossiping in trees of size $n$ is $\geq$ $3 \frac{1}{6} n-16$ and $\leq 5 n+8$.

Lower bound We use again the stack-and-knot argument, see section 2 . Consider a line $L$ of length $2 m+1$ with a knot of length 4 in the middle of the line, see Figure 6 , where $n=2 m+5$. In order to solve the gossiping problem on line $L$ all messages from the left half of $L$ have to be moved to the right half of $L$ and vice versa. All these messages have to traverse via node $a$. This requires at least $4 m$ independent time steps: $2 m$ steps in which node $a$ receives unit messages from its left and from its right neighbors, and $2 m$ steps devoted to appropriate transmissions to the left and to the right hand side. Note that line $L$ has knot $K$, and we have to guarantee that all messages are transmitted to the nodes on the knot too. Since node $b$ (in $K$ ) may interfere in the process of moving elements along line $L$, the last message $m_{x}$ transmitted through $a$ can be send in time $4 m+k$, for some $k \geq 0$. In order to complete the gossiping on knot $K$ it is still required another $m$ steps to send $m_{x}$ to the end of the line. Thus the cost of gossiping on line $L$ takes at least time $4 m+k+m=5 m+k$. On the other hand note also that during $2 m$ rounds (when $2 m$ messages originated in $L$ have been delivered to $a$ ) out of initial $4 m+k$ rounds both node $a$ and node $b$ didn't transmit. The other $2 m+k$ rounds could have been used either for transmissions from $a$ to $b$ or from $b$ towards the end of the knot $K$. Note also that the number of messages transmitted beyond $b$ in $K$ cannot exceed the number of messages delivered from $a$ to $b$. Which means that the number of messages transmitted beyond $b$ is bounded by $(2 m+k) / 2$. It means that there are at least $2 m-(2 m+k) / 2=m-k / 2$ other messages that have to be transmitted beyond $b$ in knot $K$. Due to Lemma 1 , this process requires at least $3(m-k / 2)$ additional steps. Thus to complete the gossiping task in the knot we need at least $4 m+k+3(m-k / 2)=7 m-k / 2$ time steps in total. We need to find a balance between the two bounds. They meet each other when $2 m=3 k / 2$. And this happens when $k=4 m / 3$. Thus the minimal number of steps required by gossiping on a line with one knot is $5 m+4 m / 3=6 \frac{1}{3} m \geq 3 \frac{1}{6} n-16$. Since a line with one knot is a simple form of a free tree we have proved that the gossiping problem in trees is harder than its counterpart on a line.

Upper bound We show here that the gossiping in any tree of size $n$ can be performed in time $O(n)$. We start the presentation with a simple gossiping

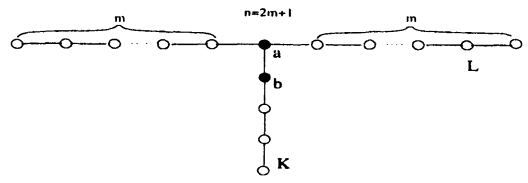

Figure 6. Line with a knot 
algorithm with running time $6 \frac{1}{2} n$, and then we present its tuned version with running time $5 n+8$.

We need the following definitions. Let $G=(V, E)$ be an undirected graph and let $r_{i}=\max _{v_{j} \in V} \operatorname{dist}\left(v_{i}, v_{j}\right)$, for each $v_{i} \in V$. A radius of $G$ is defined as $r_{c}=\min _{i=1, . .,|V|} r_{i}$ where node $v_{c}$ is called a central node. Recall also that $r_{c} \leq\lfloor n / 2\rfloor$.

Gossiping in time $\mathbf{5 n}+\mathbf{8}$ The algorithm behaves similarly to our best gossiping algorithm on a line. It collates messages in the center of the tree and simultaneously broadcasts them along its all branches. Similarly, as it happens on the line, when two sequences of messages (collated and broadcasted) work against each other, all messages are traversing with a speed $1 / 4$, i.e. one position ahead in every 4 consecutive time steps (see page 198, Process 1). However in the final stage when only the broadcast sequence is available the speed of it is much higher, and it is $1 / 2$ (see page 198 , Process 2 ). The algorithm collecting all messages in the central node can be completed in time $4(n-1)+12=4 n+8$. At this time only one message has not been broadcasted from the center (all other messages are already traversing with a speed $1 / 2$ along all branches of the tree). The time remaining to complete the gossiping process is bounded by $2 \cdot\lfloor n / 2\rfloor \leq n$, where $\lfloor n / 2\rfloor$ is the length of the longest possible branch (a radius of a tree). In total the complexity of the algorithm is bounded by $4 n+8+n=5 n+8$.

\section{General undirected graphs}

In this section we study the time complexity of gossiping in general undirected graphs.

Theorem 5 The asymptotic complexity of radio gossiping in general undirected graphs of size $n$ is $\Omega(n \log n)$ and $O\left(n \log ^{2} n\right)$.

Upper bound The algorithm is based on a broadcasting algorithm due to Chlamtac and Weinstein [5] that works in time $O\left(D \log ^{2} n\right)$, where $D$ is a diameter of the graph. Their broadcasting algorithm goes along consecutive BFS levels in the graph spending at most $O\left(\log ^{2} n\right)$ time at each level. Our gossiping algorithm runs (similarly as in trees) in two stages. In Stage 1 we collect all messages in some distinguished node $z$ transporting them (in the form of a pipeline) along branches of any BFS spanning tree rooted in $z$. This can be done in time $O(n)$. The second stage (broadcasting) is performed via pipelined distribution of $n$ unit messages from node $z$. The broadcasts are scheduled such that when the $i$ th broadcasted message is at BFS level $j$ (formed by nodes at distance $j$ from $z$ ), the wave with $(i+1)$ th broadcasted message is at level $j-3$. This allows to avoid collisions caused by messages at different BFS levels. All collisions caused by nodes on the same BFS level are handled in time $O\left(\log ^{2} n\right)$ 
using techniques from [5]. In total, the pipelined gossiping process can be accomplished in time $O\left(n \log ^{2} n\right)$.

Lower bound We need the following definitions.

Definition The undirected graph $G=\{V=L \cup R, E\}$ is proper if:

$1 G$ is bipartite, with partition $L$ and $R$,

$2|L| \leq|R|$, and

3 for each $w \in R$ there exists $v \in L$, s.t., $(v, w) \in E$.

We say that the size of a proper graph $G=\{V=L \cup R, E\}$ is $|R|$. Let $n=|R|$. A one-way broadcasting problem in proper graphs is to transmit one message originally stored in all nodes in $L$ to all nodes in $R$. A one-way gossiping problem in proper graphs is defined as follows. Assume that initially each node in $L$ has the same set $S$ of $n$ messages. The task is to deliver one copy of each of $n$ messages from $S$ to every node in $R$. (Note that it doesn't matter which node in $L$ transmits this copy)

Consider one (atomic) step of any gossiping algorithm. Two cases apply:

Case 1 Assume that there exists a proper graph $G$ with $|R|=n$, s.t., one can deliver at most $O(n / \log n)$ messages to nodes in $R$ during a single step of any communication procedure. We show that in this graph the one-way gossiping problem cannot be solved in time $o(n \log n)$. And indeed, the property of the graph imposes that the number of messages received in $R$ at any time cannot be greater than $O(n / \log n)$. Note that to accomplish one-way gossiping we need to deliver from $L$ to $R n \cdot n=n^{2}$ messages in total. However this process requires at least $n^{2} / O(n / \log n)=\Omega(n \log n)$ steps.

Update now $G$ to $G^{\prime}$, s.t., nodes in $L$ form a complete graph (to guarantee that $G^{\prime}$ is connected and gossiping in $G^{\prime}$ is feasible). We claim that gossiping in $G^{\prime}$ is harder than one-way gossiping in $G$, where set $S$ is formed by $n$ messages originated in $R$ in gossiping problem. And indeed, any algorithm that solves gossiping problem in $G$ can mimic one-way gossiping in $G^{\prime}$. This means that gossiping in $G^{\prime}$ requires $\Omega(n \log n)$ steps, and the lower bound follows in this case.

Case 2 This is a complementary case, i.e., we assume that for any proper graph there exists a way to inform in a single step $\omega(n / \log n)=n \cdot f(n) / \log n$ nodes in $R$, for some asymptotically growing positive integer function $f(n)$, s.t., $f(n)=\omega(1)$ and $f(n)=o(\log n)($ e.g. $f(n)=\sqrt{\log n})$. We show that under this assumption we can solve one-way broadcasting problem in any proper graph of size $n$ in time $o\left(\log ^{2} n\right)$.

According to our assumption, we can inform in one round at least $n$. $f(n) / \log n$ nodes in $R$. When this is done we remove all informed nodes from $R$ and select appropriate number of nodes in $L$ to much the property of proper graphs, i.e., if $|L|>|R|$ we pick at most $|R|$ nodes in $L$ to match property 3 in 
proper graphs. When this is done we perform another step of one-way broadcasting, remove informed nodes in $R$ and again select appropriate nodes in $L$. We repeat this process as long as the size of $L$ is grater than, e.g., $\log n$. Afterwards all uninformed nodes in $R$ receive broadcasting message sequentially in separate steps. Let $T(n)$ be the maximum number of time steps required by the one-way broadcasting algorithm in proper graph with $|R|=n$. The recurrence on $T(n)$ is defined as follows

$$
\begin{aligned}
& 1 T(i)=T(i-i \cdot f(i) / \log i)+1, \text { for } i>\log n, \\
& 2 T(i)=i, \text { for } i \leq \log n .
\end{aligned}
$$

In order to solve the recurrence we can find, e.g., a good estimation on a number of steps after which a value of argument $n$ will be decreased by half. Note that after each step the value of an argument is decreased by some value. Initially by value $\frac{n \cdot f(n)}{\log n}$, and after some number of steps when the value of the argument will drop to $\frac{n}{2}$, by value $\frac{\frac{n}{2} \cdot f\left(\frac{n}{2}\right)}{\log \left(\frac{n}{2}\right)}$. Since all consecutive decrements had values $\geq \frac{\frac{n}{2} \cdot f\left(\frac{n}{2}\right)}{\log \left(\frac{n}{2}\right)}$ the number of decrements can be bounded by $\frac{\frac{n}{2}}{\frac{n}{2} \cdot f\left(\frac{n}{2}\right)} \leq \frac{\log n}{\log \left(\frac{n}{2}\right)}$. Hence after at most $\frac{\log n}{2 f\left(\frac{n}{2}\right)}$ recursive steps the value of argument $n$ will be decreased by half. Thus

and

$$
T(n) \leq T\left(\frac{n}{2}\right)+\frac{\log n}{2 f\left(\frac{n}{2}\right)}
$$

$$
T(n) \leq \sum_{i=1}^{\log n} \frac{i}{2 f\left(2^{i-1}\right)} \leq \log n \cdot o(\log n)=o\left(\log ^{2} n\right)
$$

since $f(n)=o(\log n)$ and $f(n)=\omega(1)$.

This means that we can perform one-way broadcasting in any proper graph $G$ of size $n$ in time $o\left(\log ^{2} n\right)$. For any proper graph $G=\{V=L \cup R, E\}$ of size $n$ we define its extension $G^{\prime \prime}=\left\{V^{\prime \prime}, E^{\prime \prime}\right\}$, where $V^{\prime \prime}=V \cup\{\bar{v}\}$ and $E^{\prime \prime}=E \cup\{(\bar{v}, v): v \in L\}$. Note that due to our result for any $G^{\prime \prime}$ there should exist a broadcasting procedure (with source node $\bar{v}$ ) in time $o\left(\log ^{2} n\right)$. However we also know that this is not possible due to the construction of a family of graphs (extension of proper graphs) requiring $\Omega\left(\log ^{2} n\right)$ broadcasting time, by Alon et al. in [1]. This means that case 2 is not feasible and that the minimum gossiping time in undirected graphs is bounded from below by $\Omega(n \log n)$.

\section{References}

[1] N. Alon, A. Bar-Noy, N. Linial and D. Peleg, A Lower Bound for Radio Broadcast, Journal of Computer and System Sciences 43, (1991), pp. 290-298.

[2] R. Bar-Yehuda, A. Israeli, and A. Itai, Multiple communication in multi-hop radio networks, SIAM Journal on Computing 22 (1993), pp. 875-887.

[3] J.C. Bermond, L. Gargano, A.A. Rescigno, and U. Vacarro, Fast Gossiping by Short Messages, In Proc 22th Int. Colloq. on Automata, Languages and Programming, (ICALP'1995), LNCS 944, pp. 135-146. 
[4] D.P. Bertsekas and J.N.Tsitsiklis, Parallel and Distributed Computation: Numerical Methods, Prentice-Hall, Englewood Cliffs, NJ, 1988.

[5] I. Chlamtac and O. Weinstein, The Wave Expansion Approach to Broadcasting in Multihop Radio Networks, In Proc. INFOCOM, 1987.

[6] B.S. Chlebus, L. Gasieniec, A.M. Gibbons, A. Pelc, and W. Rytter, Deterministic broadcasting in unknown radio networks, In Proc. 11th ACM-SIAM Symp. on Discrete Algorithms, (SODA'2000), pp. 861-870.

[7] B.S. Chlebus, L. Gassieniec, A. Lingas, and A. Pagourtzis, Oblivious gossiping in ad-hoc radio networks, In Proc 5th Int. Workshop on Discrete Algorithms and Methods for Mobile Computing and Communications, (DIALM'2001), pp. 44-51.

[8] M. Christersson, L. Gasieniec, and A. Lingas, Gossiping with bounded size messages in ad hoc radio networks, In Proc 29th Int. Colloq. on Automata, Languages and Programming, (ICALP'2002), to appear.

[9] M. Chrobak, L. Gasieniec, and W. Rytter, Fast broadcasting and gossiping in radio networks, In Proc. 41st IEEE Symp. on Found. of Comp. Sci., (FOCS'2000), pp. 575-581.

[10] M. Chrobak, L. Gasieniec, and W. Rytter, A randomized algorithm for gossiping in radio networks, In Proc. 7th Annual Int. Computing and Combinatorics Conference, (COCOON'2001), pp. 483-492.

[11] A.E.F. Clementi, A. Monti, and R. Silvestri, Selective families, superimposed codes, and broadcasting in unknown radio networks, In Proc. 12th ACM-SIAM Symp. on Discrete Algorithms, (SODA'2001), pp. 709-718.

[12] M. Flamini and S. Perennes, Lower Bounds on the Broadcasting and Gossiping Time of Restricted Protocols, Tech Report, INRIA, January 1999.

[13] G. Fox, M. Johnson, G. Lyzenga, S. Otto, J. Salmon, and D. Walker, Solving Problems on Concurrent Processors, Volume 1, Prentice-Hall, 1988.

[14] I. Gaber and Y. Mansour, Broadcast in radio networks, in Proc. 6th Ann. ACM-SIAM Symp. on Discrete Algorithms, (SODA'1995), pp. 577-585.

[15] L. Gasieniec and A. Lingas, On adaptive deterministic gossiping in ad hoc radio networks, In Proc. 13th ACM-SIAM Symp. on Discrete Algorithms (SODA'2002), pp. 689-690.

[16] P. Indyk, Explicit constructions of selectors and related combinatorial structures, with applications, In Proc. 13th ACM-SIAM Symp. on Disc. Alg., (SODA'2002), pp. 697-704.

[17] K. Ravishankar and S. Singh, Asymptotically optimal gossiping in radio networks, Discrete Applied Mathematics 61 (1995), pp 61-82.

[18] K. Ravishankar and S. Singh, Gossiping on a ring with radios, Par. Proc. Let. 6, (1996), pp 115-126.

[19] S. Tabbane, Handbook of Mobile Radio Networks, Artech House Publishers, 2000. 